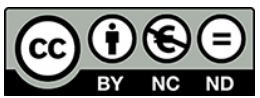

\title{
The project : how to deal with coherence and incoherence
}

\author{
Andrée Piecq \\ Master in Psychology, General Secretary UES-EUS, Honorary President of S\&O, founder of and Scientific director of \\ G.I.R.O.S (Belgium) \\ Rue Beauregard 98 / Carnières / B-7141 Belgium \\ a.piecq@skynet.be \\ Claude Lambert \\ Analyst-programmer, President of S\&O, member G.I.R.O.S. (Belgium), initiator of "Complexitude Workgroup" \\ (France) \\ 51, rue du Paquis / Halanzy / B-6792 Belgium \\ cepehello@gmail.com
}

\begin{abstract}
The project fits logically into an out of balance. From a systemic point of view, the project proposed to move structure of the system towards another structural form of the system. A change in structure of the system is called a mutation and it seems as an incoherence. In contrast, if the structure is not changed, but only the observed elements are changed, this modification is called arrangement. It operates within the system as a return to balance without changing structure and it seems coherent.

If a new structure emerges when the project is complete, the emergence of its final form appears with a lot of unpredictability, uncertainty.

The project is endangering the balance of the system, which could seem inconsistent. From this imbalance will emerge a totality whose purpose will take shape.

The project is a shock that will produce a structural bifurcation. A linear analysis of the evolution of the project is often illusory. To give coherence it is necessary to cross the levels of reality and prepare for approach steering.

The organization is a complex system and its change is considered as a meta-system that requires a meta-structure. That is to say, it must reach a meta-finality.
\end{abstract}

Keywords: project, emergence, uncertainty, incoherence, coherence, meta system, meta structure

\section{How do we understand the project?}

For decades the project is a common term that accompanies the manager and the decision maker in most organizations.

It is useful to clarify the meaning of the concept of "project" used in this article.

The project is a concept belonging to the anticipation mechanisms [1]. These anticipation mechanisms can be adaptive, cognitive, imaginary or operative. From welfare, through divination, utopia, science fiction to project concept, anticipation can take different shapes. These designs can mix conjecture, prediction or conspiracy. Classical science considers the project as if it took the form of the forecast. In the organization, although the need for anticipation can take rational forms, a common procedure is the variation in goal, in objective and in plan (action).But it can have a form of anticipation of another operating mode, fuzzy or partially determined type. This partly determined character of the project is a paradoxical concept. It means actions in the short term while incorporating the long term as conjectural. Its character, partly determined integrates with the fact that it can be only partially realized.

Our approach in this article is to consider the analysis and management of the project within the organization.

\section{Two types of projects in our approach}

With the concept of the project, we will distinguish the project that remains a planning and project that represents a break [2]. 
The implementation of projects that focus on facilities to operate within the organization is linked to notions of optimization, building, and of improvement. In other words, a project of this nature will focus on the content of information, flow changes, corrections indicators ...

It is not intended to produce a change in the structure of the system, unless it is part of a strategy of structural change and in this case it is a step.

We are interested in the project as break, as a change in organizational structure [3]. We are talking about changing organizational structure, where changes occur between the interactions that bind the foundations of system. Within this framework, the project concept comes as an anticipation undetermined.

\section{How can we consider the anticipation?}

The project is a challenge in its management; it needs to be in the same paradigm of historical and sociological contexts that define the organization. Ignoring these aspects expose the manager to the risk to the risk of a misunderstanding of the concept implemented that will lead to major differences in the management, and conduct the actors to join or not join the project.

Today the project fits in a time when the vision of progress' in parallel with the technology is in trouble as way to develop the society. Only few actors trust in a progress that fits naturally in the direction that is taking the social, the economic and the technological..

On the other hand, belief in a legacy historical and cultural of one Original Cause allows man to register on a given line is in trouble. The final cause and orientation towards a specific future is not shared by the majority of stakeholder's beliefs.

On the other hand, belief in a Original Cause, a legacy of culture and history that enabled man to lie on a given line is in trouble. The final cause and orientation towards a specific future is also no longer shared by the majority of stakeholder's belief. These divergent approaches of future and time appear at the heart of organizations become increasingly multicultural and must be taken into account without claiming to assess relevance.

The initiator of a project within an organization must take into account these differences in approaches to the concept of anticipation.

\section{Science and anticipation}

Scientific thinking qualified classical develops his theories based on a world that his scientific knowledge can anticipate in a determined way. If the future realized is inadequate with the anticipation, the logic of this conventional approach is to look for the mistake, improve the tools used to reach expectations, or even find the culprit that prevent planning. This approach may seem anachronistic but is deeply embedded in how to evaluate the project.

Cybernetics and systems replace the purpose at the heart of their approach. Systemic defined a system as interacting components transforming during time according of a project. Systemic paradigm strives to articulate the interface between finalizing purpose, the system and the environment. J.-L. Lemoigne insists on this system property to make a purposed action on the environment [4].

\section{Change and non-change}

Change can take different forms. One form is the "stability" This form of change is to change content that does not change the structure of the organization: process optimization, quality improvement, product renewal ... This form of change echoes a common understanding of the concept of innovation that give to stakeholders the freedom to propose and make changes to the content. The concept of innovation can also be applied when there is a breach of an express or implicit rule of the system. In a second step the transgression may be followed or not by its institutionalization. In both cases, it is as if the actors and subsystems are considered as independent.

But in the first case the autonomy applies to the content and does not affect the structure of the organization, in the second case it is the structure that is modified.

Change and innovation have at least two characters: a "weak" character who does not intend to change structure, a "strong" character that leads to a change in structure.

The polysemy of words creates confusion among stakeholders.

\section{Adjustment and mutation}

An adjustment will not include a change in organizational structure. It will focus on the content, the amplification process, corrections, did not affect the structure. A project, when it takes the form of a development as the environment requires a mutation is resource intensive and strengthens the functioning of the structure of the organization that you want to change making it more difficult and more complex the change. 
The mutation is a change in structure that produces the emergence of a new system consistency.

\section{Inconsistency: balance and break}

From a cultural point of view the concept of equilibrium coincides with a static approach that can become deadly if the environment in which the organization takes shape changes so that no change could lead to the disappearance of the organization. The static design and the balance of the organization is linked to the illusion to consider a system as isolated. This glance can define attributes and a limited number of indicators that are maintained over time. The organization is then viewed as a system whose structure is independent of its history.

The implementation of the project and its purpose will which is included in the finality concept discusses one paradoxical situation. Indeed, the project will create an inconsistency within the system. This instability and his paradoxical situation yet involved in the coherence of the whole is considered in a logical readings levels.

\section{Coherence and Emergence}

The project will show both consistency and inconsistency.

If the project is part of a change that the purposed willingness to go beyond the adjustment, it will introduce an inconsistency between its purpose (to go beyond the adjusting) and how behaviors of the organization were before the project start [5]. Behaviors (verbal and nonverbal) that will be observed in the new arrangement of interactions show a discrepancy with the founders of the organization elements.

During the implementation of the project, this inconsistency is reinforced by the difficulty of taking into account the difference between the result to be achieved, and the state of the organization at the time of project development. In other words it means trouble to consider what will emerge from the organization (verbal and non verbal behaviors) at the end of the project. We could say that the inconsistency reinforces the impossibility of representing the unspecified dimension of the emergence of the organization after installation of the project but also the difficulty of the language represented by the organization.

The expression of the organization in its old language will introduce inconsistencies. To avoid this trap, the project will form part of a rupture, of a mutation. The change requires the organization to the invention of a new language, to bring out a new coherence.

The construction of a new coherence becomes conscious by attention to logic levels. 


\section{The logical levels}

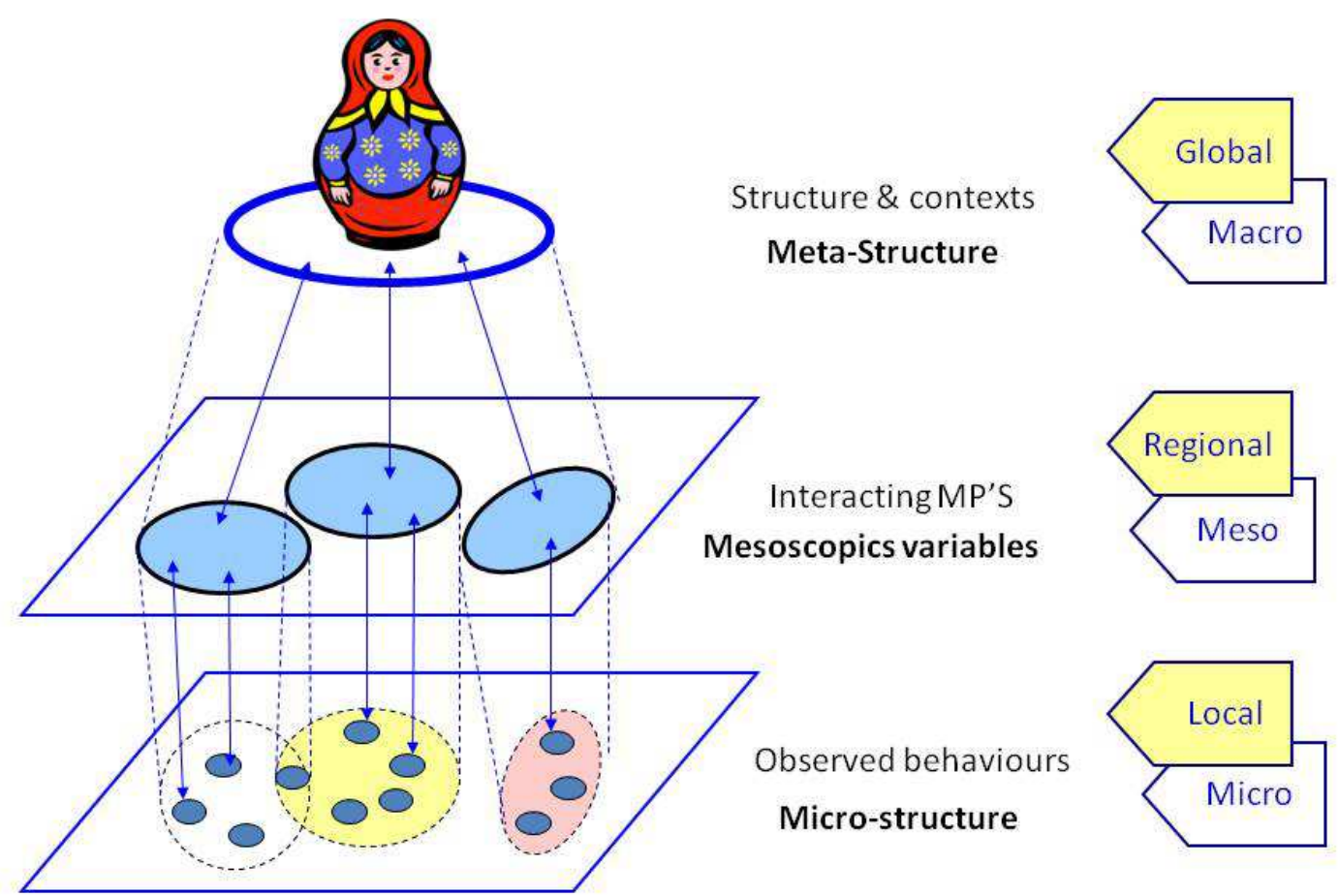

The concept of level we use to enable the invention of this new language, this new coherence refers to 3 logical levels set out in the "Giroscope."

In this article, the three levels that will help to understand the emergence of a new coherence are the:

1. Micro level regarding the interactions of contents (which is directly observable in the project and which give access to the guiding principles)

2. Meso level with respect the interactions between the guiding principles ,

3. Meta level with respect the interactions between the principles and elements of context. These elements are geographical, economic, political, cultural, linguistic, social, architectural ... and time context related to the historical context ...

Before decode what is happening in the strategy of mutation (cf. Watzlawick "Change Type II") [6] briefly look at what happens when we introduce changes that maintains consistency, while believing they will cause real change, and then establish a strategy of adjustment "Change Type I" (P.Watzlawick) [6] . 


\section{A. Adjustement}

1) Micro-level observations show that the observable behaviors belong to two very different but non separable simultaneous classes:

- The class of the descriptive contents: «Index"

- Relations (guidelines or founding principles) of asset type. : "Order"

We can make the analogy between the digital language that is the language of the content and the analogic language which is the language of the relationship. (cf. Logic of communication)

The observation of observable behaviors show:

- at the level of "Index" or "content", that behaviors take different forms (it is as if they changed)

- at the level of "order" or "relationship", they do not change and remain in the same state that we call equilibrium level.

It is as if the observable behaviors were simultaneously balance and imbalance (balance on the "order" level and imbalance in the "indice" level) which is inconsistent.

2) At the meso level, the observed behavior inconsistent cause identical interactions (guidelines do not change). Guiding principles, over time, are redundant and keep the same structure (structural isomorphic). The guiding principles are the same before and after the project. The project does not introduce structural change.

It is as if the changes observed "order" type behaviors were not canceled by the change of behavior "index" type, which does not alter the structure.

Modifications of the observed elements do not cause a new structure. The structure that emerges is the same before and after project implementation.

3) Meta-level interactions between context and guiding principles will cause a shock (see Charleroi) that regardless of class, will lead to emergence (totality principles). The project includes only descriptive elements, regardless of the structure. It does not take into account changes that the clash between contexts and guidelines interactions could occur. The interactions between guiding principles (building blocks) and the interactions between contextual elements remain in the non-change. The goal is not reached. It is as if the project has given rise to a consistent inconsistency, and the goal was not reached.

\section{B. The mutation}

1) Micro-level observation shows that (as in an adjustment) observable behaviors belong to two very different and non separable simultaneous classes:

- The class of descriptive content type Class definitions : "Index"

- Relations (guidelines or founding principles) of asset type : "Order"

The observation of observable behavior shows that

at the descriptive level we observe that the change in shape does not influence the change: it remains in equilibrium

- At the "contents" level (the descriptive level) we observe that the change in shape does not influence the change: it remains in equilibrium. It is as if the change in the shape left all in the same balance, nothing changes (which may seem paradoxical.)

But observation shows also

- At the "order" level (the active level) the definitions of relations (interactions between the different founding principles) are changed, they leave the balance.

It is as if the observable behaviors were both equilibrium and non-equilibrium and were in a paradoxical logic. It is as if there was at the same time the coherence and incoherence. 
2) Meso-level observation shows that

The paradox that exists in observable behavior leads interactions guidelines no longer be redundant. They have changed the structure changes (there is no structural isomorphism). Interactions are not identical before, during and after the project. The relationships between the guidelines are changed.

At this level of play, which was regarded as balance and change becomes inconsistent and can lead to a change in structure.

\section{3) Meta-level}

The interaction between the interactions of the elements of the context and the interactions of guiding principles will cause a shock (cf. Charleroi) leading to emergence (principle of totality).

This shock of interactions between contexts and guidelines led to a change in the structure which emerges a mutation: change of interactions between the guiding principles and contexts. This emergence is of a meta-type.

Guidelines (founding elements) at their contents may in consistency or inconsistency which paradoxically is not the change. Only interactions, how the principles are interrelated is deeply modified, the order in which they bind is different. The goal is reached and a new structure "emerges".

The mutation is in progress, it is now time to manage it.

\section{About the use of the modeling}

Within the framework of a project aimed at changing the application of existing model presents a contradiction. This renunciation of the use of existing models led us logically to a modeling approach that is understood more comprehensive than explanatory. To this end, we have equipped ourselves an approach that can evolve organizations according to the circumstances and time. This approach to modeling complex fits into the circular relationship between the project of the observer, collecting multiple observations. It leads to the progressive development of the structure of the organization by linking variables. This heuristic approach leads to the formulation of hypotheses. They allow us to diagnose the operation of the system in context. This approach is part of a circular process in which develops the consistency of a system. At the heart of our approach is built by the choice of the observer, a set of mesoscopic variables that will be powered by the field data (micro) and guided by the modeler project (macro) [7]. These mesoscopic variables are developed based on the fundamental principles of systemic. The requirement of validity of these principles is that they are devoid of ideological connotation and enough practice to facilitate the modeling work. Finally they must meet intersubjective agreement in systemic community. By analogy, the principles used play the role of operators in a complex equation that inform us on the functioning of the organization. As in mathematics, the function does not bode values, the guiding principle has no determination on the content that will be observed. The principle takes a value in relation to other principles. The weight and value of the information is not determined by a model. Of all interactions between the principles and the interactions between elements of the context emerge the structure of the organization. The emerging structure, also called meta-structure, highlights the self-organization of the system without claiming objectivity. This model does not pretend to completeness of representation and is based on the incompleteness considered as a resource. Modeling the organization seen as emergence allows us to act to maintain or change the properties of the system depending on the application.

\section{Conclusion}

The project is part of a qualified form of anticipation fuzzy in the sense of the impossibility of determining in advance the gap with the expected result. The control model does not account for the emergence. We propose the approach of modeling tools in which the variables are constructed by observation. The approach by the Giroscope involves a tiered approach: a micro-level observation of behavior, a meso level of development of the structure of interactions between the guidelines and finally a macro level with the clash between the structure and elements of context. This approach can accommodate the coexistence of consistency and inconsistency manifested in the steering of project. Piloting is part of a circular process evaluation.

\section{References}

[1] Jean-Pierre Boutinet, “Anthropologie du Projet”, PUF, Paris,1990, pp 49-81

[2] P.Watzlawick, J.Weakland, R.Fisch, "Changements", Points, 1975

[3] Andrée Piecq, "De la pensée systémique à la pratique de l'organisation, Le giroscope”, Paris : L'Harmattan, 2011

[4] J.-L. Lemoigne, « Théorie du système général », France : mcxapc, 2006

[5] Andrée Piecq, Claude Lambert, « How to develop an “open” future: Is it possible to take advantage of coherence and incoherence?", Vienna : EMCSR,

[7] Minati, G. and Licata, I., "Emergence as Mesoscopic Coherence", Systems, 1(4), 50-65, 2013 